\title{
Further Bisabolenes and Dammarane Triterpenes of Commiphora kua Resin
}

\author{
Lawrence Onyango Arot MAnguro, ${ }^{*, a}$ Ivar UGI, ${ }^{b}$ and Peter Lemmen ${ }^{b}$ \\ ${ }^{a}$ Chemistry Department, Nairobi University; P. O. Box 30197, Nairobi, Kenya: and ${ }^{b}$ Technische Universitaet Muenchen, \\ Institut fuer Organische Chemie und Biochemie; Lehrstuhl 1, Lichtenbergstrasse 4, 85747-Garching, Germany. \\ Received July 15, 2002; accepted December 27, 2002
}

From the resins of Commiphora kua a novel bisabolene; 6-hydroxy-2-methyl-5-(5'-hydroxy-1'(R),5'-dimethylhex-3'-enyl)-phenol together with two new dammarane triterpenes, $3 \beta, 16 \beta, 20(S), 25$-tetrahydroxydammar-23-ene and $3 \beta$-acetoxy-16 $\beta, 20(S), 25$-trihydroxydammar-23-ene, have been isolated. In addition, being reported are known compounds identified as 2-methyl-5- $\left(4^{\prime}(S)\right.$-hydroxy-1'(R),5'-dimethylhex-5'-enyl)-phenol, 2acetoxyfuranodienone, 2 -methoxyfuranodienone, $3 \beta, 16 \beta, 20(R)$-trihydroxydammar-24-ene and its acetate derivative, $3 \beta$-acetoxy-16 $\beta, 20(R)$-dihydroxydammar-24-ene, and $\beta$-amyrin and its acetate derivative. 2-Methyl-5-(4' $(S)$ hydroxy-1'(R),5'-dimethylhex-5'-enyl)-phenol displayed fungicidal activity against Cladosporium cucumernum on TLC assay.

Key words aromatic sesquiterpene; dammarane triterpene; Commiphora kua; Burseraceae

The plant species Commiphora kua (Burseraceae) is known for an oleoresin exudate which is an important article of commerce. ${ }^{1)}$ The plant is also used in herbal medicine as a remedy for snakebite, gonorrhoea, stomach disorders, and livestock related diseases. ${ }^{2}$

Previous phytochemical studies on the plant resin afforded cycloartane triterpenes, ${ }^{3)}$ bisabolenes, and furanosesquiterpenoids based on a germacrane skeleton, ${ }^{4)}$ dammarane triterpenes, and octanordammaranes. ${ }^{5)}$ In continuing with our study, we now report the isolation and structural elucidation of bisabolene; 6-hydroxy-2-methyl-5-(5'-hydroxy-1' $(R), 5^{\prime}$-dimethylhex-3'-enyl)-phenol (1) along with two novel dammarane triterpenes; $3 \beta, 16 \beta, 20(S), 25$-tetrahydroxydammar-23-ene (3) and its acetate derivative, $3 \beta$-acetoxy-16 $\beta, 20(S), 25$-trihydroxydammar-23-ene (4). Also being reported from the plant are known compounds characterised as 2-methyl-5-(4' $(S)$-hydroxy-1' $(R), 5^{\prime}$-dimethylhex-5' -enyl)-phenol (2), ${ }^{6)} 2$-methoxyfuranodienone and 2-acetoxyfuranodienone, ${ }^{7)} \beta$-amyrin and its acetate derivative, ${ }^{8)} 3 \beta, 16 \beta, 20(R)$-trihydroxydammar-24ene (5) and its acetate, $3 \beta$-acetoxy-16 $\beta, 20(R)$-dihydroxydammar-24-ene (6). ${ }^{9)}$ Their structures have been established based on extensive spectroscopic (UV, IR, ${ }^{1} \mathrm{H}$ - and ${ }^{13} \mathrm{C}-\mathrm{NMR}$ and MS) and chemical studies.

Compound $\mathbf{2}$ inhibited the growth of the plant pathogenic fungus Cladosporium cucumerinum on thin layer chromatography plates.

\section{Results and Discussion}

Compound 1 displayed a molecular ion peak at $\mathrm{m} / \mathrm{z} 250$ in the mass spectrum, corresponding to $\mathrm{C}_{15} \mathrm{H}_{22} \mathrm{O}_{3}$. In its ${ }^{1} \mathrm{H}-$ NMR spectrum the main features of a tetra-substituted benzene ring were evident: two low field protons appearing as doublets at $\delta_{\mathrm{H}} 6.90$ and 6.70 , a benzenoid methyl singlet $\left(\delta_{\mathrm{H}}\right.$ $2.23)$, a benzylic proton $\left(\delta_{\mathrm{H}} 2.60\right)$, and two benzenoid hydroxyls $\left(\delta_{\mathrm{H}} 7.20, \mathrm{D}_{2} \mathrm{O}\right.$ exchangeable $)$ were present. The two dimensional total correlation spectroscopy (2D TOCSY) experiments ${ }^{10,11)}$ together with ${ }^{1} \mathrm{H}-{ }^{1} \mathrm{H}$ correlation spectroscopy (COSY) correlations were used to identify the spin system for the two protons, thus locating them at positions C-3 and C-4, respectively, a fact further corroborated by nuclear
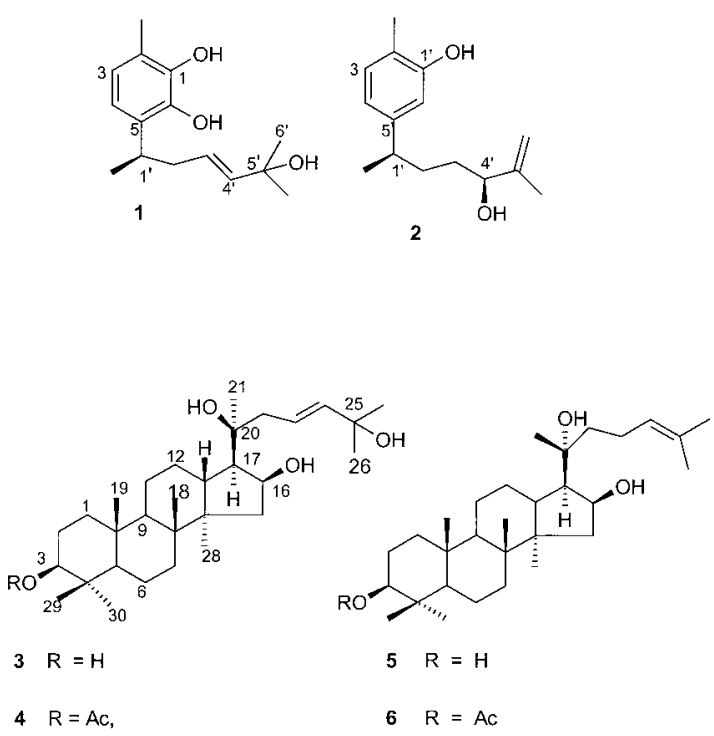

Overhauser effect spectroscopy (NOESY) cross peaks (Fig. $1)$. The structure of the $5^{\prime}$-hydroxy-1' $(R), 5^{\prime}$-dimethylhex- $3^{\prime}$ enyl side chain at $\mathrm{C}-5$ was confirmed by ${ }^{1} \mathrm{H}$ - and ${ }^{13} \mathrm{C}-\mathrm{NMR}$, ${ }^{1} \mathrm{H}$-detected heteronuclear multiple quantum coherence (HMQC) and heteronuclear multiple bond connectivity (HMBC) experiments. ${ }^{12}$ )

Also characteristic of the ${ }^{1} \mathrm{H}-\mathrm{NMR}$ spectrum were the presence of a hydroxy isopropyl moiety at $\delta_{\mathrm{H}} 1.30$ and a twoproton multiplet at $\delta_{\mathrm{H}} 5.56$. The latter resolved in benzene- $d_{6}$ indicating the presence of a trans double bond. ${ }^{6)}$ The ${ }^{13} \mathrm{C}$ NMR data (Table 1) ascertained these molecular features and peaks at $\delta_{\mathrm{C}} 72.6\left(\mathrm{C}-5^{\prime}\right), 124.7\left(\mathrm{C}-3^{\prime}\right)$, and $138.2\left(\mathrm{C}-4^{\prime}\right)$ were in accordance with the foregoing evidence. Furthermore, the two carbons at $\delta_{\mathrm{C}} 124.7$ and 138.2 represented an internal double bond and were linked to protons at $\delta_{\mathrm{H}} 5.50$ and 5.46, respectively. The HMBC correlations (Fig. 1) confirmed the double bond to be between $\mathrm{C}-3^{\prime}$ and $\mathrm{C}-4^{\prime}$ and both the olefinic proton peaks correlated with $\delta_{\mathrm{C}} 72.6\left(\mathrm{C}-5^{\prime}\right)$.

The configuration at $\mathrm{C}-1^{\prime}$ was established as $R$ from the cd spectrum which exhibited a positive Cotton effect at $320 \mathrm{~nm}$ 

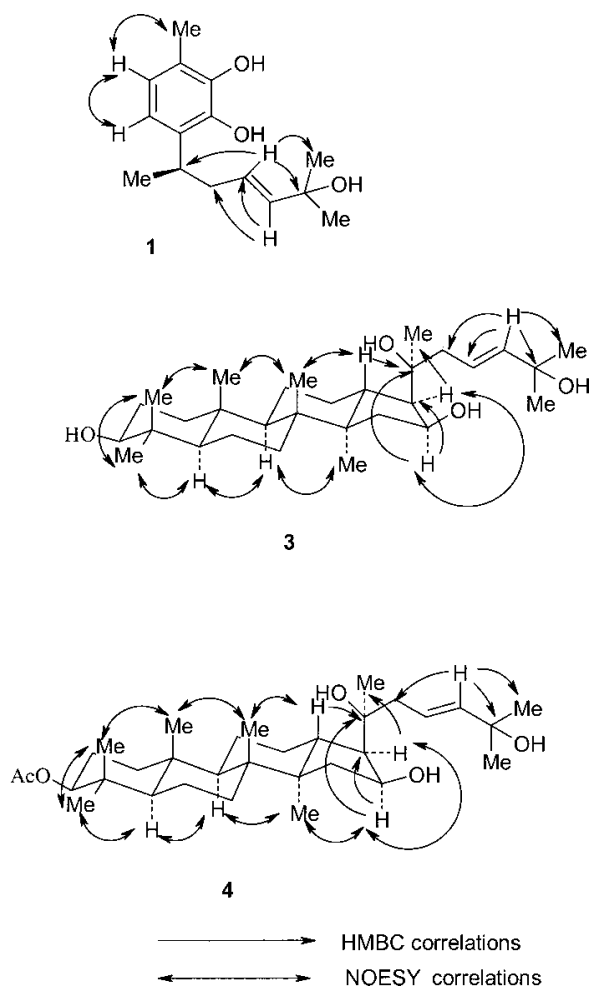

Fig. 1. Pertinent Correlations in the HMBC and NOESY Spectra for Compounds 1, 3 and 4

( $\Delta \varepsilon$ 3.6). This was further supported by comparison of the ${ }^{1} \mathrm{H}-\mathrm{NMR}$ data with those reported on similar curcumene derivatives. ${ }^{13,14)}$ On the basis of the spectroscopic evidence accrued, the structure of compound 1 was established as 6-hydroxy-2-methyl-5-(5'-hydroxy-1' $(R), 5^{\prime}$-dimethylhex-3' enyl)-phenol.

Compound 2, a yellow oil, showed a molecular ion peak at $m / z 234$ corresponding to $\mathrm{C}_{15} \mathrm{H}_{22} \mathrm{O}_{2}$. Unlike compound 1 described above, its ${ }^{1} \mathrm{H}$ - and ${ }^{13} \mathrm{C}$-NMR spectral data suggested a trisubstituted benzene ring with a monounsaturated side chain at C-5. ${ }^{15)}$ In its mass spectrum, a characteristic McLafferty process involving the elimination of the side chain produced an ion at $m / z 148\left[\mathrm{M}-\mathrm{C}_{5} \mathrm{H}_{10} \mathrm{O}\right]^{+}$and another one at $m / z 135\left[\mathrm{M}-\mathrm{C}_{6} \mathrm{H}_{10} \mathrm{O}\right]^{+}$, both fragments were consistent with the presence of a secondary hydroxyl function. ${ }^{16)}$

The complete ${ }^{1} \mathrm{H}$ - and ${ }^{13} \mathrm{C}-\mathrm{NMR}$ chemical shift assignments for $\mathbf{2}$ were deduced from concerted application of ${ }^{1} \mathrm{H}-{ }^{1} \mathrm{H}$ COSY and ${ }^{1} \mathrm{H}$ detected 'one bond' and long range $(\mathrm{C}$, $\mathrm{H})$ correlation experiments as well as comparison with already reported data on the compound. ${ }^{6}$ In a ${ }^{1} \mathrm{H}-{ }^{1} \mathrm{H}$ homonuclear chemical shift correlation spectrum, the allylic carbinol proton at $\delta_{\mathrm{H}} 4.20$ showed connectivities with two other protons, thus limiting the position of the hydroxyl group to C-4'. The one bond proton-carbon chemical shift correlation was established using the HMQC sequence while the assignment of quartenary carbons was obtained from the analysis of long range correlation responses over three bonds using an HMBC technique. The absolute configuration at C-4' was established as $S$ by the GC modification of the Horeau method, ${ }^{17)}$ which afforded an excess product of $(R)-\alpha$ phenylbutyric acid (65\% optical yield) as identified by GC and GC-MS analysis. This was further supported by the cd spectrum positive Cotton effect at $246 \mathrm{~nm}(\Delta \varepsilon$ 2.6) and the
Table $1 .{ }^{13} \mathrm{C}$-NMR of Compounds $\mathbf{1}-\mathbf{5}$

\begin{tabular}{|c|c|c|c|c|c|}
\hline Carbon & 1 & 2 & 3 & 4 & 5 \\
\hline 1 & 142.0 & 154.2 & 38.2 & 39.1 & 38.6 \\
\hline 2 & 122.0 & 121.0 & 27.4 & 30.5 & 34.5 \\
\hline 3 & 123.5 & 130.4 & 75.0 & 76.4 & 73.4 \\
\hline 4 & 118.3 & 118.8 & 40.0 & 39.6 & 40.1 \\
\hline 5 & 131.4 & 146.8 & 55.3 & 56.0 & 55.5 \\
\hline 6 & 141.4 & 114.0 & 19.5 & 19.2 & 20.3 \\
\hline $\mathrm{CH}_{3}-2$ & 18.7 & 20.4 & & & \\
\hline $1^{\prime}$ & 39.3 & 40.0 & & & \\
\hline $2^{\prime}$ & 37.5 & 36.0 & & & \\
\hline $3^{\prime}$ & 124.7 & 32.9 & & & \\
\hline $4^{\prime}$ & 138.2 & 77.2 & & & \\
\hline $5^{\prime}$ & 72.6 & 147.3 & & & \\
\hline $6^{\prime}$ & 29.4 & 112.4 & & & \\
\hline $\mathrm{Me}-1^{\prime}$ & 22.0 & 21.8 & & & \\
\hline $\mathrm{Me}-5^{\prime}$ & 30.1 & 17.0 & & & \\
\hline \multicolumn{6}{|l|}{ OAc- $4^{\prime}$} \\
\hline \multicolumn{6}{|l|}{ Me-Ac } \\
\hline 7 & & & 35.3 & 34.7 & 35.0 \\
\hline 8 & & & 41.2 & 40.6 & 40.3 \\
\hline 9 & & & 51.3 & 50.5 & 50.4 \\
\hline 10 & & & 36.5 & 37.0 & 37.0 \\
\hline 11 & & & 22.0 & 21.6 & 22.0 \\
\hline 12 & & & 27.1 & 26.8 & 27.0 \\
\hline 13 & & & 51.1 & 49.0 & 51.6 \\
\hline 14 & & & 52.0 & 50.3 & 48.2 \\
\hline 15 & & & 43.2 & 41.0 & 44.0 \\
\hline 16 & & & 74.6 & 74.4 & 74.0 \\
\hline 17 & & & 42.0 & 41.7 & 39.4 \\
\hline 18 & & & 18.3 & 17.7 & 18.3 \\
\hline 19 & & & 16.0 & 15.8 & 16.3 \\
\hline 20 & & & 76.3 & 77.30 & 76.0 \\
\hline 21 & & & 25.9 & 26.3 & 26.0 \\
\hline 22 & & & 34.4 & 35.2 & 43.5 \\
\hline 23 & & & 127.5 & 127.6 & 22.7 \\
\hline 24 & & & 138.4 & 140.3 & 124.6 \\
\hline 25 & & & 81.6 & 81.3 & 131.6 \\
\hline 26 & & & 25.3 & 26.3 & 25.8 \\
\hline 27 & & & 25.0 & 24.8 & 18.3 \\
\hline 28 & & & 19.0 & 18.3 & 18.9 \\
\hline 29 & & & 22.2 & 21.5 & 19.7 \\
\hline 30 & & & 14.0 & 15.2 & 15.8 \\
\hline OAc & & & & 170.4 & \\
\hline$\underline{\mathrm{Me}}-\mathrm{Ac}$ & & & & 21.0 & \\
\hline
\end{tabular}

${ }^{1} \mathrm{H}-\mathrm{NMR}$ coupling constant $J_{3^{\prime}-4^{\prime}}=6.8,4.4 \mathrm{~Hz} .{ }^{13}$ )

From these spectral data, the structure of 2 was deduced to be 2-methyl-5-(4' $(S)$-hydroxy-1' $(R), 5^{\prime}$-dimethylhex-5' -enyl)phenol.

The other component (3) considered novel afforded a molecular ion peak in the MS at $m / z$ 476, calculated to be $\mathrm{C}_{30} \mathrm{H}_{52} \mathrm{O}_{4}$. From a preliminary observation, its mass spectrum exhibited the main features of a dammarane triterpene ${ }^{9)}$ as evidenced by fragment peaks at $\mathrm{m} / \mathrm{z}$ 376, 315, 208, and 207. The ${ }^{13} \mathrm{C}$-NMR spectrum showed a total of 30 carbons and their multiplicity assignments using DEPT established 8 methine, 8 methylene, and 8 methyl groups, and 6 quartenary carbon atoms were identified from the difference of the broad band. The HMQC, HMBC, and NOESY experiments were employed in assigning all the ${ }^{1} \mathrm{H}$ and ${ }^{13} \mathrm{C}$ values and by comparison with the respective spectral data for compound $\left.\mathbf{5},{ }^{9}\right)$ it differed from the latter only in the side chain composition. According to the ${ }^{1} \mathrm{H}$ - and ${ }^{13} \mathrm{C}$-NMR data, the side chain of $\mathbf{3}$ consisted of two $\mathrm{OH}$ groups on substituted quartenary carbons $\left(\mathrm{C}-20, \delta_{\mathrm{C}} 76.3\right.$ and $\left.\mathrm{C}-25, \delta_{\mathrm{C}} 81.6\right)$, two germinal 
methyls $\left(\mathrm{C}-26\right.$ and $\mathrm{C}-27, \delta_{\mathrm{H}} 1.28$, with respective $\delta_{\mathrm{C}} 25.3$ and 25.0), and a double bond (C-23 and C-24, $\delta_{\mathrm{H}} 5.40$ and $5.50, \delta_{\mathrm{C}} 127.5$ and 138.4). The structure of the side chain was confirmed from HMBC correlations (Fig. 1) which showed cross peaks between the proton signals of H-17 $\left(\delta_{\mathrm{H}}\right.$ $2.20)$, and $\mathrm{H}-22\left(\delta_{\mathrm{H}} 2.40\right)$ with the carbon signal of C-20 $\left(\delta_{\mathrm{C}}\right.$ 76.3). Furthermore, the H-24 signal $\left(\delta_{\mathrm{H}} 5.50\right)$ exhibited long range coupling with carbon signals of $\mathrm{C}-22\left(\delta_{\mathrm{C}} 34.4\right), \mathrm{C}-23$ $\left(\delta_{\mathrm{C}} 127.5\right), \mathrm{C}-26\left(\delta_{\mathrm{C}} 25.3\right)$, and $\mathrm{C}-27\left(\delta_{\mathrm{C}} 25.0\right)$.

The stereochemistry of the chiral carbon C-20 sustaining $\mathrm{OH}$ group in equatorial orientation was defined on the basis of the cd spectrum in $\mathrm{MeOH}$ results which gave a positive Cotton effect at $276 \mathrm{~nm}(\Delta \varepsilon 2.2)$ that closely resembled that of guggulsterol (274 nm, $\Delta \varepsilon 2.6)$ previously isolated from Indian Commiphora mukul. ${ }^{18)}$ Thus, compound 3 was deduced to be $3 \beta, 16 \beta, 20(S), 25$-tetrahydroxydammar-23-ene.

The last compound (4) showed eight tertiary methyl groups at $\delta 1.33,1.20,1.06,1.03,0.98,0.90$, and 0.88 in the ${ }^{1} \mathrm{H}-$ NMR spectrum. The ${ }^{13} \mathrm{C}$-NMR exhibited 32 carbon resonances. Distortionless enhancement by polarization transfer (DEPT) experiments suggested the presence of eight methylenes, eight methines, nine methyls, and 7 quartenary carbon atoms were identified from the difference of the broad band. The HMQC, HMBC, and NOESY experiments were similarly employed in assigning all the ${ }^{1} \mathrm{H}$ and ${ }^{13} \mathrm{C}$ values and by comparison with the respective data for 3 , compound 4 differed from the former only in the acetate peak. The stereochemistry at C-20 with an $\mathrm{OH}$ group in an equatorial orientation was similarly determined to be $S$ using cd spectrum positive Cotton effect at $278 \mathrm{~nm}(\Delta \varepsilon 1.8)$, which was similar to that of compound 3 . These findings confirmed that $\mathbf{4}$ is an acetate derivative of $\mathbf{3}$ and therefore was identified as $3 \beta$-acetoxy-16 $\beta, 20(S), 25$-trihydroxydammar-23-ene.

\section{Experimental}

General Melting points (uncorrected) were determined on a Buchi 535 melting point apparatus. IR spectra were run as $\mathrm{KBr}$ disc on an infrared IMR-25 spectrophotometer. The electron impact (EI)-MS were measured on a $70 \mathrm{eV}$ MAT $8200 \mathrm{~A}$ Varian MAT Bremen instrument. ${ }^{1} \mathrm{H}$ - and ${ }^{13} \mathrm{C}-\mathrm{NMR}$ spectra were recorded on a Brucker WM NMR spectrometer operating at 400 and $100 \mathrm{MHz}$, respectively, and were recorded in $\mathrm{CDCl}_{3}$ using tetramethylsilane (TMS) as an internal standard. $[\alpha]_{D}$ values were obtained on a JASCO DIP-360 instrument. Capillary GC was performed on a Carlo-Erba Mega instrument, $25 \mathrm{~m} \times 0.33 \mathrm{~mm}$ SE-30 column, initial temperature $70^{\circ} \mathrm{C}$ (2 min), then rising at $5{ }^{\circ} \mathrm{C} / \mathrm{min}$ to $100^{\circ} \mathrm{C}$ followed by $10^{\circ} \mathrm{C} / \mathrm{min}$ to a final temperature of $250^{\circ} \mathrm{C} ; \mathrm{N}_{2}$ at $20 \mathrm{~cm} / \mathrm{s}$. Preparative GC was performed on a $3 \mathrm{~m} \times 2 \mathrm{~mm}$ i.d. Carbowax $20 \mathrm{M}$ packed column operated at $180^{\circ} \mathrm{C}$. GC-MS was obtained on a Hewlett-Packard 5890/5988A instrument in the EI mode $(70 \mathrm{eV})$ connected to a 100E-series data processing system. The SE-30 column $(12 \mathrm{~m} \times 0.33 \mathrm{~mm})$, was operated under the following conditions; initial temperature $60^{\circ} \mathrm{C}(2 \mathrm{~min})$, and final temperature $200^{\circ} \mathrm{C}$, rate $5^{\circ} \mathrm{C} / \mathrm{min}, \mathrm{He}$ at $25 \mathrm{~cm} / \mathrm{s}$.

Resin Samples Samples were collected from trees in the Isiolo District on the main road to Wajir, Kenya in February 1996. Botanical samples including leaves, branches and pods were sampled and voucher specimens (NTR/KEFRI 96/2/CK) were deposited at the Kenya Forestry Research Institute Herbarium. Samples were stored in airtight bags and kept in a refrigerator until studied.

Extraction and Isolation The residue from steam distilled resin was extracted in the cold with EtOAc $(1.01 \times 2)$ and evaporated under reduced pressure to give $40 \mathrm{~g}$ of a dark brown gummy material. A portion of the residue $(35 \mathrm{~g})$ was chromatographed over a silica gel column, eluted first with $n$-hexane followed by the same solvent containing increasing amounts of $\mathrm{CH}_{2} \mathrm{Cl}_{2}, \mathrm{EtOAc}$, and finally with $\mathrm{MeOH}$. One hundred milliliter fractions of each were collected. A total of 150 fractions were sampled and their composition monitored by silica gel TLC in two systems; A ( $n$-hexane-EtOAc $4: 1,2: 1$ and $1: 1)$ and $\mathrm{B}\left(\mathrm{CH}_{2} \mathrm{Cl}_{2}-\mathrm{MeOH}, 9: 1\right)$. The spots were visualised by spraying with acidified anisaldehyde reagent and heating. The corresponding eluates were combined into pools (I-IV).

Pool I (fractions 15-40, 5.5 g) upon low pressure column chromatography with $n$-hexane-EtOAc $(4: 1)$ followed by the same solvent system in the ratio $3: 1$ allowed the isolation of $\beta$-amyrin and its acetate derivative, ${ }^{8)} 2$ methoxyfuranodienone, and 2-acetoxyfuranodienone. ${ }^{7)}$ Pool II (fractions $41-80,4.5 \mathrm{~g})$ afforded a viscous brown resin and was similarly purified using flash chromatography over a silica gel column with $n$-hexane-EtOAc $(3: 1,3: 2), 150$ fractions of $20 \mathrm{ml}$ each being collected. This procedure gave $\mathbf{1}(35.4 \mathrm{mg}), \mathbf{2}(65.5 \mathrm{mg}), \mathbf{4}(55.3 \mathrm{mg})$, and $\mathbf{6}(85 \mathrm{mg})$. Pool III (fractions $81-$ $112,5.5 \mathrm{~g}$ ) using a similar technique as in pool II yielded $75 \mathrm{mg}$ of $\mathbf{3}$, a further $33.4 \mathrm{mg}$ of $4,52.3 \mathrm{mg}$ of $\mathbf{5}$, and a further $26.6 \mathrm{mg}$ of $\mathbf{1}$.

Pool IV (fractions 115-150, 7.5 g) was rechromatographed over a $2 \%$ oxalic acid deactivated silica gel column with $\mathrm{CH}_{2} \mathrm{Cl}_{2}-\mathrm{MeOH}(9: 1)$ to give an unidentified sugar compound $(300 \mathrm{mg})$.

Identification of Compounds The structures of known compounds were identified by comparison of their physical and spectroscopic data with those of known standards.

Compound 1: Yellow oil $[\alpha]_{\mathrm{D}}^{25}-35^{\circ}\left(c=1.0, \mathrm{CH}_{2} \mathrm{Cl}_{2}\right)$. UV $\lambda_{\max }(\mathrm{MeOH})$ $274(\log \varepsilon 2.90) \mathrm{nm} . \mathrm{CD}(c=0.5, \mathrm{MeOH}): 320(\Delta \varepsilon 3.6) \mathrm{nm} . \mathrm{IR}(\max (\mathrm{KBr})$ $3450,1620,1250$, and $1080 \mathrm{~cm}^{-1}$. ${ }^{1} \mathrm{H}-\mathrm{NMR}\left(\mathrm{CDCl}_{3}\right) \delta_{\mathrm{H}}: 7.20(2 \mathrm{H}, \mathrm{s}, \mathrm{OH}-1$ and $\mathrm{OH}-6, \mathrm{D}_{2} \mathrm{O}$ exchang. $), 6.90\left(1 \mathrm{H}, \mathrm{d}, J_{\mathrm{AB}}=7.8 \mathrm{~Hz}, \mathrm{H}-3\right), 6.70(1 \mathrm{H}, \mathrm{d}$, $\left.J_{\mathrm{AB}}=7.8 \mathrm{~Hz}, \mathrm{H}-4\right), 5.56\left(2 \mathrm{H}, \mathrm{m}, \mathrm{H}-3^{\prime}, \mathrm{H}^{\prime} 4^{\prime}\right), 2.60(1 \mathrm{H}, \mathrm{tq}, J=6.7,6.7$ $\left.\mathrm{Hz}, \mathrm{H}-1^{\prime}\right), 2.40\left(2 \mathrm{H}, \mathrm{m}, \mathrm{CH}_{2}-2^{\prime}\right), 2.23(3 \mathrm{H}, \mathrm{s}, \mathrm{Me}-2), 1.35(3 \mathrm{H}, \mathrm{d}, J=$ $\left.7 \mathrm{~Hz}, \mathrm{Me}-1^{\prime}\right), 1.30\left(6 \mathrm{H}, \mathrm{s}, \mathrm{Me}_{2}-5^{\prime}\right) .{ }^{1} \mathrm{H}-\mathrm{NMR}\left(\mathrm{C}_{6} \mathrm{D}_{6}\right) \delta_{\mathrm{H}}: 7.02(1 \mathrm{H}, \mathrm{d}$, $J=7.5 \mathrm{~Hz}, \mathrm{H}-3), 6.97(2 \mathrm{H}, \mathrm{s}, \mathrm{OH}-1, \mathrm{OH}-6), 6.85(1 \mathrm{H}, \mathrm{d}, J=7.5 \mathrm{~Hz}, \mathrm{H}-4)$, $5.50\left(1 \mathrm{H}\right.$, ddd, $\left.J=15.5,6.5,6 \mathrm{~Hz}, \mathrm{H}-3^{\prime}\right), 5.46\left(1 \mathrm{H}\right.$, br d, $\left.J=15.5 \mathrm{~Hz}, \mathrm{H}-4^{\prime}\right)$, $2.70\left(1 \mathrm{H}, \mathrm{tq}, J=6.9,6.9 \mathrm{~Hz}, \mathrm{H}-1^{\prime}\right), 2.10(3 \mathrm{H}, \mathrm{s}, \mathrm{Me}-2), 1.25(3 \mathrm{H}, \mathrm{d}$, $\left.J=6.6 \mathrm{~Hz}, \mathrm{Me}-1^{\prime}\right), 1.20\left(6 \mathrm{H}, \mathrm{s}, \mathrm{Me}_{2}-5^{\prime}\right) \cdot{ }^{13} \mathrm{C}$-NMR data: see Table 1. Hreims $[\mathrm{M}]^{+} 250.2909$ (Calcd for $\left.\mathrm{C}_{15} \mathrm{H}_{22} \mathrm{O}_{3}, 250.2916\right)$. EI-MS $(70 \mathrm{eV}): \mathrm{m} / \mathrm{z}(\%)$ 250 (6), 234 (10), 233, 217 (14), 174 (35), 152 (26), 141 (100), 137 (28), and 41 (27).

Compound 2: Yellow oil. $[\alpha]_{\mathrm{D}}^{25}-13.5^{\circ}\left(c=1.0, \mathrm{CH}_{2} \mathrm{Cl}_{2}\right)$. UV $\lambda_{\max }$ $(\mathrm{MeOH}) 275(\log \varepsilon 2.85) \mathrm{nm} . \mathrm{CD}(c=0.5, \mathrm{MeOH}) 324(\Delta \varepsilon 2.90)$ and 246 $(\Delta \varepsilon 4.2) \mathrm{nm}$. IR $v_{\max }(\mathrm{KBr}) 3400,1610,1450,1250,870 \mathrm{~cm}^{-1} .{ }^{1} \mathrm{H}-\mathrm{NMR}$ $\left(\mathrm{CDCl}_{3}\right) \delta_{\mathrm{H}}: 7.01(1 \mathrm{H}, \mathrm{d}, J=10.7 \mathrm{~Hz}, \mathrm{H}-3), 6.80(1 \mathrm{H}, \mathrm{d}, J=10.7 \mathrm{~Hz}, \mathrm{H}-4)$, $6.40(1 \mathrm{H}, \mathrm{s}, \mathrm{H}-6), 4.90\left(1 \mathrm{H}, \mathrm{s}, \mathrm{H}-6_{\mathrm{B}}{ }^{\prime}\right), 4.85\left(1 \mathrm{H}, \mathrm{s}, \mathrm{H}-6_{\mathrm{A}}{ }^{\prime}\right), 4.20(1 \mathrm{H}, \mathrm{dd}$, $\left.J=6.8,4.4 \mathrm{~Hz}, \mathrm{H}-4^{\prime}\right), 2.65\left(1 \mathrm{H}, \mathrm{tq}, J=6.6,6.6 \mathrm{~Hz}, \mathrm{H}-1^{\prime}\right), 2.40(3 \mathrm{H}, \mathrm{s}, \mathrm{Me}-2)$, $1.80\left(2 \mathrm{H}, \mathrm{m}, \mathrm{CH}_{2}-2^{\prime}\right), 1.50\left(2 \mathrm{H}, \mathrm{m}, \mathrm{CH}_{2}-3^{\prime}\right), 1.65\left(3 \mathrm{H}, \mathrm{s}, \mathrm{Me}-5^{\prime}\right), 1.20(3 \mathrm{H}$, d, $\left.J=6.0 \mathrm{~Hz}, \mathrm{Me}-1^{\prime}\right) .{ }^{13} \mathrm{C}$-NMR data: see Table 1. EI-MS $(70 \mathrm{eV}): \mathrm{m} / \mathrm{z}(\%)$ $\mathrm{M}^{+} 234$ (20), 216 (11), 215 (3), 201 (10), 174 (5), 159 (18), 148 (100), 135 (50), $115(80)$.

Compound 3: Colourless needles, $[\alpha]_{\mathrm{D}}^{25}+13.6^{\circ}\left(c=1.0, \mathrm{CH}_{2} \mathrm{Cl}_{2}\right)$. CD $(c=0.5, \mathrm{MeOH}): 276(\Delta \varepsilon 2.2) \mathrm{nm}$. IR $v_{\max }(\mathrm{KBr}) 3500,1640 \mathrm{~cm}^{-1} .{ }^{1} \mathrm{H}-$ NMR $\delta_{\mathrm{H}}: 5.50(1 \mathrm{H}, \mathrm{dd}, J=14.5,7.5 \mathrm{~Hz}, \mathrm{H}-24), 5.40(1 \mathrm{H}, \mathrm{ddd}, J=14.5,7.5$, $7 \mathrm{~Hz}, \mathrm{H}-23), 4.60(1 \mathrm{H}$, ddd, $J=8.5,7.6,5.2 \mathrm{~Hz}, \mathrm{H}-16), 3.75(1 \mathrm{H}, \mathrm{dd}, J=10.5$, $4.4 \mathrm{~Hz}, \mathrm{H}-3), 2.50\left(1 \mathrm{H}\right.$, ddd, $\left.J=15.6,9.7,7.5 \mathrm{~Hz}, \mathrm{H}-2_{\mathrm{ax}}\right), 2.45(1 \mathrm{H}$, ddd, $\left.J=15.6,8.0,6.4 \mathrm{~Hz}, \mathrm{H}-2_{\mathrm{eq}}\right), 2.40(1 \mathrm{H}, \mathrm{m}, \mathrm{H}-22), 2.20$ (1H, ddd, $J=12.5,7.6$, $4.2 \mathrm{~Hz}, \mathrm{H}-17), 2.05\left(3 \mathrm{H}, \mathrm{s}, \mathrm{CH}_{3} \mathrm{CO}^{-}\right), 1.83\left(1 \mathrm{H}, \mathrm{dd}, J=14.3,7.1 \mathrm{~Hz}, \mathrm{H}-15_{\mathrm{eq}}\right)$, $1.28\left(6 \mathrm{H}, \mathrm{s}, \mathrm{Me}_{2}-25\right), 1.15,1.08,1.01,0.96,0.93,0.88(18 \mathrm{H}, 6 \times \mathrm{Me}) .{ }^{13} \mathrm{C}-$ NMR data: see Table 1. Hreims $[\mathrm{M}]^{+} 476.3026$ (Calcd for $\mathrm{C}_{30} \mathrm{H}_{52} \mathrm{O}_{4}$, 476.3032). EI-MS: $m / z(\%) 476(2), 458\left[\mathrm{M}-\mathrm{H}_{2} \mathrm{O}\right]^{+}(5), 440\left[\mathrm{M}-2 \mathrm{H}_{2} \mathrm{O}\right]^{+}$ (30), 433 (11), 376 (20), 359 (8), 315 (15), 298 (4), 255 (25), 226 (6), 208 (16), 207 (8), 109 (43).

Compound 4: Colourless needles, $[\alpha]_{\mathrm{D}}^{25}+38.3^{\circ}\left(c=0.5, \mathrm{CH}_{2} \mathrm{Cl}_{2}\right), \mathrm{mp}$ $140-142^{\circ} \mathrm{C} . \mathrm{CD}(c=0.5, \mathrm{MeOH}): 278(\Delta \varepsilon 1.8 \mathrm{~nm})$. IR $v_{\max }(\mathrm{KBr}) 3400$, $1735,1375,1075$ and $1040 \mathrm{~cm}^{-1} .{ }^{1} \mathrm{H}-\mathrm{NMR} \delta_{\mathrm{H}}: 5.71(1 \mathrm{H}, \mathrm{m}, \mathrm{H}-24), 5.60$ $(1 \mathrm{H}, \mathrm{m}, \mathrm{H}-23), 4.55(1 \mathrm{H}, \mathrm{dd}, J=8.6,4.6 \mathrm{~Hz}, \mathrm{H}-3), 4.45(1 \mathrm{H}, \mathrm{ddd}, J=7.9,7.7$, $5.3 \mathrm{~Hz}, \mathrm{H}-16), 2.45\left(1 \mathrm{H}, \mathrm{ddt}, J=15.7,10,7 \mathrm{~Hz}, \mathrm{H}-2_{\mathrm{ax}}\right), 2.0(1 \mathrm{H}, \mathrm{ddd}$, $\left.J=14.8,7.3,5.0 \mathrm{~Hz}, \mathrm{H}-2_{\mathrm{eq}}\right), 2.60(1 \mathrm{H}, \mathrm{d}, J=11,6.5 \mathrm{~Hz}, \mathrm{H}-13), 1.83(1 \mathrm{H}, \mathrm{dd}$, $J=12.1,7.2 \mathrm{~Hz}, \mathrm{H}-17), 1.67$ ( $\left.1 \mathrm{H}, \mathrm{dd}, J=12.8,7 \mathrm{~Hz}, \mathrm{H}-15_{\mathrm{eq}}\right), 1.55$ (1H, dd, $\left.J=13.7,5.4 \mathrm{~Hz}, \mathrm{H}-15_{\mathrm{ax}}\right), 2.40\left(2 \mathrm{H}, \mathrm{m}, \mathrm{CH}_{2}-22\right), 1.33\left(6 \mathrm{H}, \mathrm{s}, \mathrm{Me}_{2}-25\right), 1.20$, $1.06,1.03,0.98,0.9,0.88(18 \mathrm{H}, \mathrm{s}, 6 \times \mathrm{Me}) .{ }^{13} \mathrm{C}-\mathrm{NMR}$ data: see Table 1. Hreims $[\mathrm{M}]^{+} 518.3736$ (Calcd. for $\left.\mathrm{C}_{32} \mathrm{H}_{54} \mathrm{O}_{5}, 518.3818\right)$. EI-MS: $\mathrm{m} / \mathrm{z}(\%)$ $518(2), 500\left[\mathrm{M}-\mathrm{H}_{2} \mathrm{O}\right]^{+}(2), 482\left[\mathrm{M}-2 \mathrm{H}_{2} \mathrm{O}\right]^{+}(4), 457(5), 452(10), 419$ (15), 402 (7), 357 (11), 341 (20), 249 (8), 208 (30), 192 (10), 189 (60), 109 (70), $43(100)$.

Compound 5: Colourless needles, mp 210-212 ${ }^{\circ} \mathrm{C},[\alpha]_{\mathrm{D}}^{25}-20^{\circ}(c=0.60$, $\mathrm{CH}_{2} \mathrm{Cl}_{2}$ ). IR $v_{\max }(\mathrm{KBr}) 3400,1625 \mathrm{~cm}^{-1} .{ }^{1} \mathrm{H}-\mathrm{NMR} \delta_{\mathrm{H}}: 5.14(1 \mathrm{H}, \mathrm{t}, J=7 \mathrm{~Hz}$, $\mathrm{H}-24), 4.45(1 \mathrm{H}$, ddd, $J=8.5,7.6,5.3 \mathrm{~Hz}, \mathrm{H}-16), 3.50(1 \mathrm{H}, \mathrm{dd}, J=10.4$, $5.3 \mathrm{~Hz}, \mathrm{H}-3), 1.28,1.25,1.05,0.98,0.88,0.86,0.80,0.75(24 \mathrm{H}, \mathrm{s}, 8 \times \mathrm{Me})$. ${ }^{13} \mathrm{C}-\mathrm{NMR}$ data: see Table 1. EI-MS M ${ }^{+} 460$ (2), $442\left[\mathrm{M}-\mathrm{H}_{2} \mathrm{O}\right]^{+}$(34), 424 $\left[\mathrm{M}-2 \mathrm{H}_{2} \mathrm{O}\right]^{+}(18), 360$ (11), $341(20), 315$ (16), 207 (33), 135 (65), 121 (30), 109 (57), 95 (45), 81 (42), 71 (20), 69 (100). 
Assignment of C-4' Stereochemistry for Compound 2 To the compound $(10 \mathrm{mg})$ dissolved in dry pyridine $(1 \mathrm{ml})$ was added excess of $( \pm)-\alpha$ phenylbutyric anhydride ${ }^{17)}$ and the mixture was allowed to stand at room temperature under stirring. After $24 \mathrm{~h}$ the reaction mixture was added to ice$\mathrm{H}_{2} \mathrm{O}$, extracted with $\mathrm{Et}_{2} \mathrm{O}$, and dried $\left(\mathrm{Na}_{2} \mathrm{SO}_{4}\right)$ and evaporated under reduced pressure. The residue obtained was subjected to $\mathrm{GC}$ analysis and the results showed an excess of $(R)$ - $\alpha$-phenylbutyric acid ( $t_{\mathrm{R}} 14 \mathrm{~min}, 65 \%$ optical yield), an esterification product which was further confirmed with preparative GC and GC-MS analyses.

Fungicidal Test A suspension of $C$. cucumerinum prepared in a medium as recommended by Homan and Fuchs ${ }^{19}$ was sprayed on developed thin layer chromatograms containing crude extract at varying concentrations. The plates were incubated in a moist atmosphere for $24 \mathrm{~h}$ at room temperature. Clear inhibition zones (white spots) on observed TLC plates confirmed the presence of an active compound in the extract. Fractionation of the crude extract afforded compound $\mathbf{2}$ as the active ingredient.

Acknowledgements The authors thank the Alexander von Humboldt Foundation for awarding L. Manguro a fellowship. The African Academy of Sciences (AAS) is also acknowledged for a research grant. Dr. Rudolf Hermann of Technische Universitaet Muenchen, Germany is thanked for providing the NMR and MS data. Professor Ermias Dagne of Addis Ababa University, Ethiopia, is acknowledged for the cd data.

\section{References and Notes}

1) Trease G. E., Evans W. C., "Pharmacognosy," 11th ed., Balliere Tindall, London, 1988, pp. $463-477$.

2) Kokwaro J. O., "Medicinal Plants of East Africa," East African Literature Bureau, Nairobi, 1976, pp. 76-85.
3) Provan G. J., Waterman P. G., Phytochemistry, 27, 3841-3843 (1988).

4) Manguro L. O. A., Mukonyi K. M., Githiomi J. K., Planta Med., 32, $85-87$ (1996).

5) Provan P. J., Waterman P. G., Phytochemistry, 22, 917-922 (1983).

6) Aguilar M. I., Delago G., Bye R., Linares E., Phytochemistry, 33, $1161-1163$ (1993).

7) Brieskorn C. H., Noble P., Phytochemistry, 22, 87-91 (1983).

8) Ahmad V. V., Ali A., Ali Z., Bagai F. T., Zafar F. N., Phytochemistry, 49, 829-834 (1998).

9) Fattorusso E., Santacrose C., Xaasan F. C., Phytochemistry, 24, 1035-1037 (1985).

10) Greisinger C., Otting G., Wurtrich K., Ernest R. R., J. Am. Chem. Soc., 110, 7870-7875 (1984).

11) Kessler K., Oschkinat H., Greisinger C., Bermel W., J. Mag. Reson., 70, 106-110 (1986).

12) Verotta L., Orsini F., Tato M., El-Sebakhy N. A., Toima S. M., Phytochemistry, 49, 845-852 (1998).

13) Buttler M. S., Capon R. J., Nadeson R., Beverridge A. A., J. Nat. Prod., 54, 619-623 (1991).

14) Joseph-Nathan P., Toar-Miranda R., Martinenez E., Santillan R. I., J. Nat. Prod., 51, 1116-1128 (1988).

15) Akhtar N., Malik A., Afza N., Badar Y., J. Nat. Prod., 56, 295-299 (1993).

16) Hasan C. M., Islam A., Ahmed M., Mofizud D., Waterman P. G., Phytochemistry, 23, 2587-2590 (1984).

17) Brooks C. J. W., Gilbert J. D., Chem. Commun., 1973, 194-198 (1973).

18) Patil V. D., Nayak U. R., Dev S., Tetrahedron, 28, 2341-2352 (1972).

19) Homan A. L., Fuchs A., J. Chromatogr, 51, 327-329 (1970). 